\title{
ATMOSPHERIC AEROSOL MEASUREMENTS BY EMPLOYING A POLARIZATION SCHEIMPFLUG LIDAR SYSTEM
}

\author{
Liang Mei ${ }^{1 *}$, Peng Guan ${ }^{1}$, \& Yang Yang ${ }^{1}$ \\ ${ }^{1}$ School of Optoelectronic Engineering and Instrumentation Science, Dalian University of Technology, \\ Dalian 116024,China, *meiliang@dlut.edu.cn
}

\begin{abstract}
A polarization Scheimpflug lidar system based on the Scheimpflug principle has been developed by employing a compact 808 -nm multimode highpower laser diode and two highly integrated CMOS sensors in Dalian University of Technology (DLUT), Dalian, China. The parallel and orthogonal polarized backscattering signal are recorded by two 45 degree tilted image sensors, respectively. Atmospheric particle measurements were carried out by employing the polarization Scheimpflug lidar system.
\end{abstract}

\section{INTRODUCTION}

Lidar (light detection and ranging) techniques have been widely employed for atmospheric remote sensing of particles/aerosols and gases since decades. Conventional lidar techniques measure atmospheric backscattering echoes based on the time-of-flight principle, by employing high-power nanoscale lasers and photomultiplier tubes (PMT) [1-5]. Since 1980s, polarization lidar, capable of detecting the parallel and orthogonal polarized backscattering light respectively, has been employed as an effective tool for the studies of atmospheric cloud phase, ice crystals, and particle classification [6-9], etc. Nevertheless, high power pulsed Nd:YAG lasers and PMTs are to some extent complicated and of high cost. Besides, both analog and single photon counting (SPC) detection schemes are often utilized to increase the dynamic range as the lidar signal decreases over the square of the distance. All these high technical demands increase the system cost, complexity and maintenance requirement, which limit the applications of conventional pulsed lidar techniques mainly in scientific fields.

Recently, a new atmospheric lidar technique, referred to as Scheimpflug lidar, is developed for atmospheric gas and aerosol monitoring [10-13]. The Scheimpflug lidar technique is based on the Scheimpflug principle, which describes the relationship between the object, image and lens planes when they are non-paralleled in an imaging system. Briefly, if a collimated laser beam is transmitted into atmosphere, the entire laser beam over km-range can be clearly imaged by a titled image sensor while using a large aperture lens/telescope if the image sensor plane, the laser beam plane and the lens plane intersect with each other. The atmospheric backscattering signal can then be measured, and the sensor pixel corresponds to the distance. The range-resolved lidar signal is in this case angular-resolved, while it is time-resolved in conventional pulsed lidar techniques [10]. The fundamental difference of the Scheimpflug lidar technique enables the usage of low-cost, compact highpower continuous-wave laser diodes and highlyintegrated $\mathrm{CMOS} / \mathrm{CCD}$ image sensors, which greatly reduces the system complexity and cost.

In this work, we demonstrate a polarization Scheimpflug lidar system for the polarization property studies of atmospheric aerosols. A ground-based polarization Scheimpflug lidar system, based on lowcost, compact laser diodes and CMOS sensors, was developed in Dalian University of Technology, China. Atmospheric remote sensing measurements have been performed by using the polarization Scheimpflug lidar system to validate the system feasibility.

\section{METHODOLOGY}

The system schematic of the polarization Scheimpflug lidar is shown in Figure 1. A 4 W $808 \mathrm{~nm}$ multimode ( $3 \mathrm{~nm}$ bandwidth) laser diode is employed as light source. The laser beam is collimated by a F6 achromatic telescope (Focal length $600 \mathrm{~mm}, \emptyset 100 \mathrm{~mm}$ ) for transmission. A half-wave plate is placed before the achromatic lens for rotating the polarization of the laser beam, which can be used to calibrate the polarizationdependent system constant. The atmospheric backscattering light is collected by a F4 Newtonian telescope (focal length $800 \mathrm{~mm}, \emptyset 200 \mathrm{~mm}$ ). The received backscattering light first passes through an absorption filter (RG780, Edmund Optics) and an interference filter (808 nm, $3 \mathrm{~nm}$ FWHM, Edmund 
Optics) before separated by a polarization beam splitter cube. Two titled CMOS sensors (CMOSIS, $2088 \times 1088$ pixels, $5.5 \mu \mathrm{m}$ pixel width) are utilized to simultaneously detect the orthogonal and parallel polarized backscattering light. A trigger output signal from one of the CMOS sensor is fed to a Johnson counter, which then generate a square signal to modulate the laser diode for dynamic background subtraction. The equipment mentioned above are first assembled and then carried by the two arms of an equatorial mount (Skywatcher, EQ6) in accordance with the Scheimpflug principle.

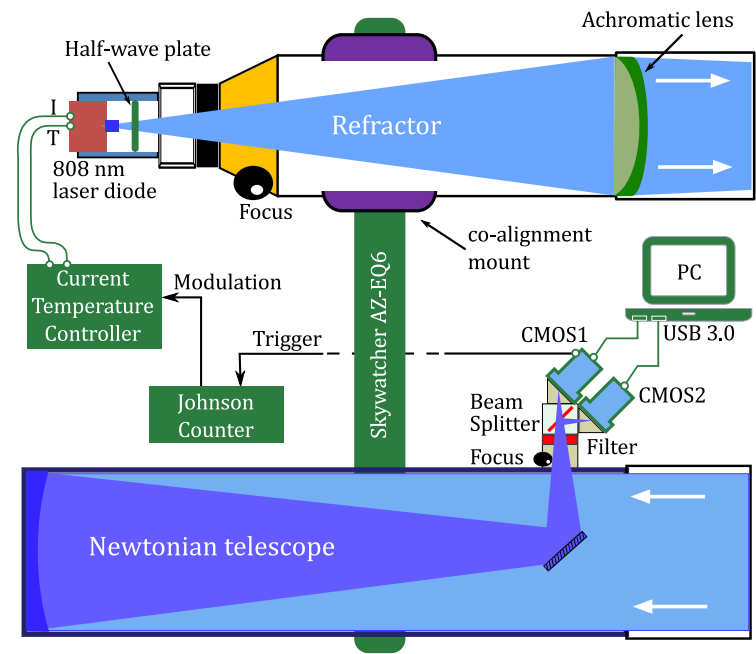

Figure 1. The schematic of the Scheimpflug Lidar system. The co-alignment mount is capable of tuning the refractor in two dimensions for the field-of-view overlap between the Newtonian telescope and the refractor. The half-wave plate can be rotated manually.

The laser beam image recorded by the CMOS sensor after subtraction of a background image is vertically (perpendicular to the laser beam transmission direction) binned in a LabVIEW-based program developed by our research group. The pixel binning process leads to a single pixel-intensity curve. After the system calibration with a hard target at a known distance [10], the pixel-distance relationship can be calculated and the distance-intensity curve, i.e., the range-resolved lidar signal is then retrieved. According to the Scheimpflug principle and geometrical optics, the lidar signal detected by the Scheimpflug lidar technique is given by [10]

$$
P(\lambda, z)=K \beta(\lambda, z) \exp \left[-2 \int_{0}^{z} \alpha\left(\lambda, z^{\prime}\right) d z^{\prime}\right] .
$$

Here, $\lambda$ is the laser center wavelength, $z$ is the measurement distance, $\beta(\lambda, z)$ and $\alpha(\lambda, z)$ are the backscatter and extinction coefficients at the distance $z$. From the above equation, we note that the lidar signal does not decrease over the square of the distance which is the case in conventional pulsed lidar techniques. This greatly facilitates the detection scheme of the Scheimpflug lidar technique, and improves the signal-to-noise ratio (SNR) for the far distance. Following Eq. (1), the depolarization ratio $\delta(z)$ can be given by [14]

$$
\delta(z)=\frac{k_{p}}{k_{s}} \frac{P_{s}(\lambda, z)}{P_{p}(\lambda, z)}=\mathrm{k} \frac{P_{s}(\lambda, z)}{P_{p}(\lambda, z)} .
$$

Here $P_{p}(\lambda, z)$ and $P_{s}(\lambda, z)$ are the parallel and orthogonal polarized signal. $k_{p}$ and $k_{s}$ are the system constant in the parallel and orthogonal channels of the Scheimpflug lidar system, and $k=k_{p} / k_{s}$ is a polarization-dependent system constant of the Scheimpflug lidar system. The system constant $k$ can be calibrate by, e.g., non-polarized light source method, air molecular method and half wave-plate method [15]. In this article, we employ the half wave-plate method to determine the system constant $k$ during the measurement campaign.

\section{MEASUREMENTS AND RESULTS}

Depolarization measurements of atmospheric aerosol were performed on December $14^{\text {th }}, 2016$ from 12:30 - 21:00. The local temperature was about $-6^{\circ}$ to $-3^{\circ}$. The laser beam was pointed to the south. Atmospheric backscattering signal was measured with $20 \mathrm{~ms}$ exposure time, and each lidar curve was generated from the mode-average of 5000-time recordings (about 4 minutes). The time-range maps of the parallel and orthogonal polarized light are shown in Figure 2. The system constant factor was calibrated by the half-wave plate method. Since atmospheric backscattering signal is angular resolved for the Scheimpflug lidar technique, the system constant factor was found to be variable for different pixels/distances in this case. In the present work, an averaged value of the polarization dependent system constant was estimated, i.e., 1.3. The Depolarization ratio map can then be retrieved. The high depolarization ratio, observed in Figure 3 , should be attributed to the highway maintenance at around 200-300 $\mathrm{m}$ which 
generated significant amount of dust, and the wind from north (Level 3-4).
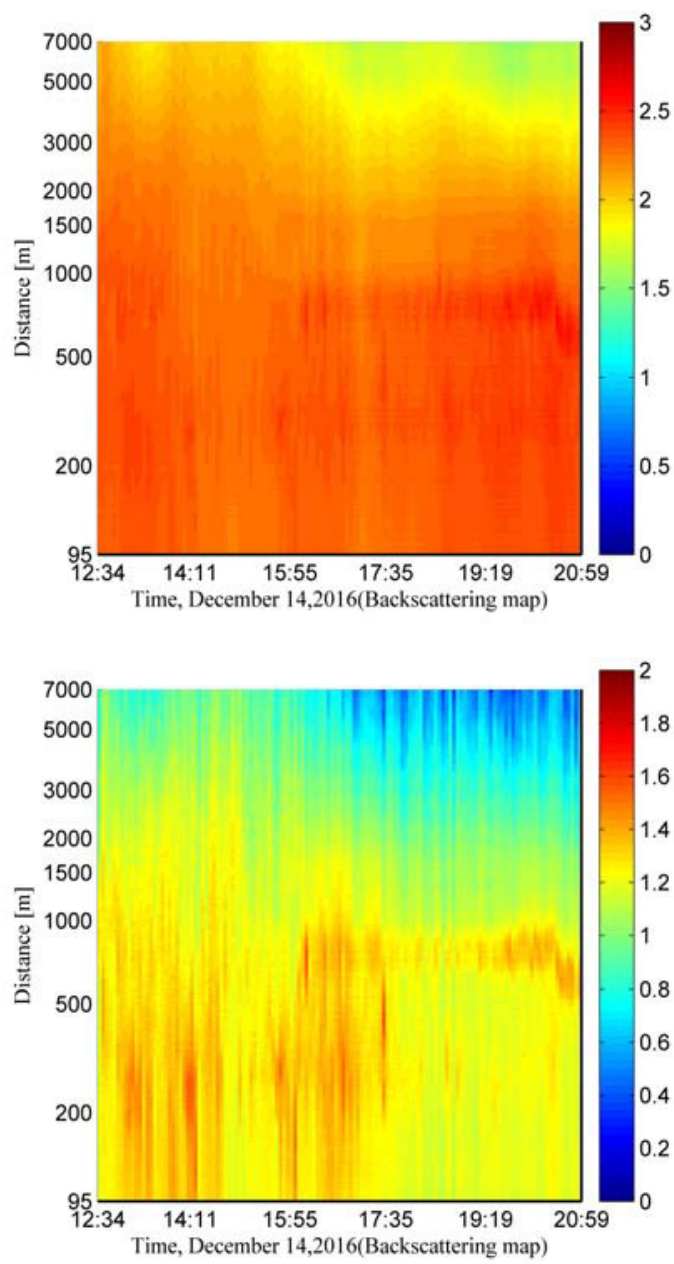

Figure 2. Parallel (up) and orthogonal (Bottom) polarized atmospheric backscattering time - range map measured by the Scheimpflug lidar system.

\section{CONCLUSIONS}

A polarization Scheimpflug lidar system as well as the preliminary results were presented in this work. As has been demonstrated, atmospheric depolarization ratio can be retrieved over severalkilometer range by the Scheimpflug lidar technique even during day time. Detailed analysis about the system constant calibration accuracy, atmospheric vertical measurements will be presented in the near future to further validate the capability of the polarization Scheimpflug lidar technique.

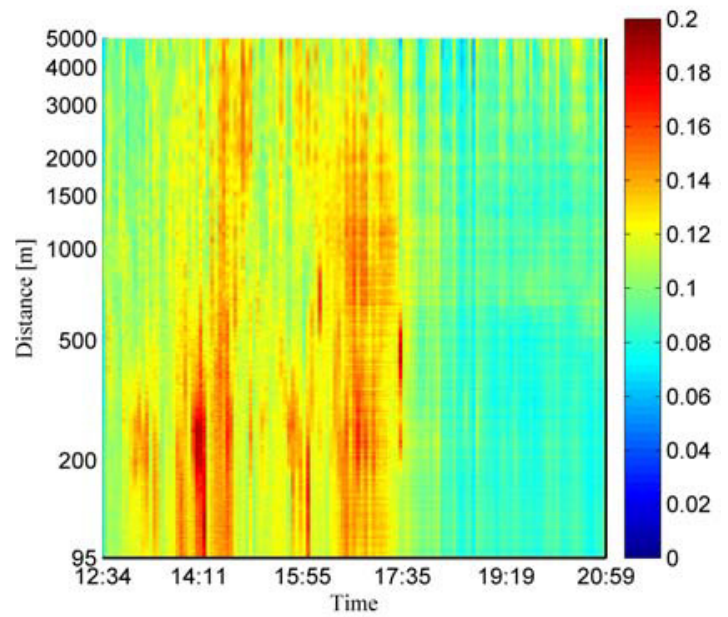

Figure 3. Depolarization ratio time - range map measured by the Scheimpflug lidar system.

\section{ACKNOWLEDGEMENTS}

National key research and development program of China (2016YFC0200600); Fundamental Research Funds for the Central Universities (DUT15RC(3)107); Natural Science Foundation of Liaoning Province, China (201602163) .

\section{References}

[1] M. Sicard, F. Molero, J. L. Guerrero-Rascado, R. Pedros, F. J. Exposito, C. Cordoba-Jabonero, J. M. Bolarin, A. Comeron, F. Rocadenbosch, M. Pujadas, L. Alados-Arboledas, J. A. MartinezLozano, J. P. Diaz, M. Gil, A. Requena, F. NavasGuzman, and J. M. Moreno, 2009: Aerosol lidar intercomparison in the framework of SPALINETThe Spanish lidar network:methodology and results, IEEE Trans. Geoscience and Remote Sensing 47, 3547-3559.

[2] Z. G. Guan, P. Lundin, L. Mei, G. Somesfalean, and S. Svanberg, 2010: Vertical lidar sounding of atomic mercury and nitric oxide in a major Chinese city, Appl. Phys. B 101, 465-470.

[3] J. Lenoble, L. Remer, and D. Tanre, Aerosol Remote Sensing. (Springer, Verlag Berlin Heidelberg, 2013).

[4] L. Mei, G. Y. Zhao, and S. Svanberg, 2014: Differential absorption lidar system employed for background atomic mercury vertical profiling in south China, Opt. Laser Eng. 55, 128-135.

[5] G. Pappalardo, A. Amodeo, A. Apituley, A. Comeron, V. Freudenthaler, H. Linne, A. Ansmann, J. Bosenberg, G. D'Amico, I. Mattis, L. Mona, U. Wandinger, V. Amiridis, L. Alados-Arboledas, D. 
Nicolae, and M. Wiegner, 2014: EARLINET: towards an advanced sustainable European aerosol lidar network, Atmos. Meas. Tech. 7, 2389-2409.

[6] S. Pal and A. Carswell, 1978: Polarization properties of lidar scattering from clouds at $347 \mathrm{~nm}$ and 694 nm, Appl. Opt. 17, 2321-2328.

[7] V. Noel, D. M. Winker, M. McGill, and P. Lawson, 2004: Classification of particle shapes from lidar depolarization ratio in convective ice clouds compared to in situ observations during CRYSTAL - FACE, Journal of Geophysical Research: Atmospheres 109,

[8] J. A. Shaw, N. L. Seldomridge, D. L. Dunkle, P. W. Nugent, L. H. Spangler, J. J. Bromenshenk, C. B. Henderson, J. H. Churnside, and J. J. Wilson, 2005: Polarization lidar measurements of honey bees in flight for locating land mines, Opt. Exp. 13, 58535863.

[9] S. S. Matthew Hayman, and Bruce Morley, 2014: Polarization lidar observations of backscatter phase matrices from oriented ice crystals and rain, Opt. Exp. 22, 16976-16990.

[10] L. Mei and M. Brydegaard, 2015: Atmospheric aerosol monitoring by an elastic Scheimpflug lidar system, Opt. Exp. 23, 247841.

[11] L. Mei and M. Brydegaard, 2015: Continuouswave differential absorption lidar, Laser \& Photon. Rev. 9, 629-636.

[12] P. Guan and L. Mei, "Range-resolved atmospheric aerosol monitoring by a Scheimpflug lidar system based on a continuous-wave laser diode," Asia Communications and Photonics Conferene (ACP), Wuhan, China, AS1F.5, (2016).

[13] L. Mei and M. Brydegaard, "Development of a Scheimpflug lidar system for atmospheric aerosol monitoring," EPJ Web of Conferences (ILRC 27), 119, 27005, (2016).

[14] K. Sassen, "Polarization in lidar," in Lidar, edited by C.Weitkamp (Springer, New York, 2005), pp. $19-42$.

[15] Z. Wang, R. Chi, B. Liu, and J. Zhou, 2008: Depolarization properties of cirrus clouds from polarization lidar measurements over Hefei in spring, Chinese Optics Letters 6, 235-237. 